East African Medical Journal Vol. 80. No 8 August 2003

QUALITY OF GLYCAEMIC CONTROL IN AMBULATORY DIABETICS AT THE OUT-PATIENT CLINIC AT KENYATTA NATIONAL HOSPITAL, KENYA C.F. Otieno, MBChB, MMed, Lecturer, Department of Medicine, College of Health Sciences, University of Nairobi, P. O. Box 19676, Nairobi, Kenya, M. Kariuki, MBChB, MMed (Ophthal), Department of Ophthalmology, Kenyatta National Hospital, P.O. Box 20723, Nairobi, Kenya and L. Ng'ang'a, PhD, Senior Research Officer, Kenya Medical Research Institute, P. O. Box 54840, Nairobi, Kenya

Request for reprints to: Dr. C.F. Otieno, Department of Medicine, College of Health Sciences, University of Nairobi, P.O. Box 19676, Nairobi, Kenya

\title{
QUALITY OF GLYCAEMIC CONTROL IN AMBULATORY DIABETICS AT THE OUT-PATIENT CLINIC OF KENYATTA NATIONAL HOSPITAL, NAIROBI
}

\author{
C.F. OTIENO, M. KARIUKI and L. NG'ANG'A
}

\begin{abstract}
Background: Treatment of diabetes mellitus is based on the evidence that lowering blood glucose as close to normal range as possible is a primary strategy for reducing or preventing complications or early mortality from diabetes. This suggests poorer glycaemic control would be associated with excess of diabetes-related morbidity and mortality. This presumption is suspected to reach high proportions in developing countries where endemic poverty abets poor glycaemic control. There is no study published on Kenyan patients with diabetes mellitus about their glycaemic control as an audit of diabetes care.

Objective: To determine the glycaemic control of ambulatory diabetic patients.

Design: Cross-sectional study on each clinic day of a randomly selected sample of both type I and 2 diabetic patients.

Setting: Kenyatta National Hospital.

Methods: Over a period of six months, January 1998 to June 1998. During routine diabetes care in the clinic, mid morning random blood sugar and glycated haemoglobin (HbAI c)were obtained.

Results: A total of 305 diabetic patients were included, $52.8 \%$ were females and $47.2 \%$ were males. 58.3\% were on Oral Hypoglycaemic Agent (OHA) only, 22.3\% on insulin only; 9.2\% on OHA and insulin and $4.6 \%$ on diet only. 39.5\% had mean HbAlc $\leq 8 \%$ while $60.5 \%$ had HbAlc $\geq 8 \%$. Patients on diet-only therapy had the best mean HbAlc $=7.04 \%$ while patients on OHA-only had the worst mean HbAlc $=9.06 \%$. This difference was significant $(\mathrm{p}=0.01)$. The former group, likely, had better endogenous insulin production. The influence of age, gender and duration of diabetes on the level of glycaemic control observed did not attain statistically significant proportions.

Conclusion: The majority of ambulatory diabetic patients attending the out-patient diabetic clinic had poor glycaemic control. The group with the poorest level of glycaemic control were on OHA-only, while best control was observed amongst patients on diet-only, because of possible fair endogenous insulin production. Poor glycaemic control was presumed to be due to sub-optimal medication and deteriorating diabetes. There is need to empower patients with knowledge and resources to enhance their individual participation in diabetes self-care. Diabetes care providers and facilities also need capacity building to improve care of patients with diabetes.
\end{abstract}

\section{INTRODUCTION}

Diabetes mellitus is emerging rapidly as a major public health problem in the developing countries, both in numbers (1) and costs of management (2).

Treatment of diabetes is based on the rationale that lowering the blood sugar to near normal range is a primary strategy that reduces and/or prevents complications and/or early mortality and morbidity $(3,4)$. These mega-studies of DCCT and UKPDS addressed type 1 and 2 diabetic populations respectively, with fairly uniform message on glycaemic control. However, multicentre trials of such magnitude may not discriminate well the issues related to patients, care centers and the health care providers that affect attainment of the desired glycaemic goals, which also parallel the glycaemic levels achieved.
Poor glycaemic control as a major cause of morbidity and mortality in diabetes is presumed to reach high proportions in developing countries where resources are scarce. It is also important to note that while UKPDS (4) suggested that blood pressure (and lipid control in Type 2 disease) may be of greater importance than glycaemic control, the latter is still an important measure of diabetes care. In Kenya like many other developing countries of Africa, organised diabetes care is lacking within the existing health care delivery systems, supplies and skilled manpower are scarce. Insulin and oral hypoglycaemic agents are very costly both to governments and individual patients. Where organised diabetes care is available, it is largely inaccessible to many patients due to distance, high cost of travel and the care itself. 
Kenyatta National Hospital, the larger teaching and national referral hospital in Kenya, is host to about 3500 patients with diabetes mellitus each year (5), most of them attending the outpatient clinic or may be hospitalised to receive in-patient care for various reasons. Many of these patients are seen in the clinic at very long intervening intervals. However, lately, improvements have been made to enable diabetic patients with special individual needs to be reviewed more frequently by the nurses and clinical officers at the clinic.

This study was conducted on an unselected population of ambulatory diabetic patients to determine the quality of glycaemic control attained in this outpatient clinic as an audit of diabetics care thereof.

\section{MATERIALS AND METHODS}

Design: This was a cross-sectional study conducted in the diabetic outpatient clinic of Kenyatta National Hospital. After approval by the Ethical Review Committee of the hospital, the study was conducted over a six-month period between January 1998 and June 1998. Patients were not notified of their potential inclusion in the study.

During the six-month period, a total of 305 patients were randomly selected on each weekly clinic day and included in the study after informed verbal consent was obtained. This represented about $35 \%$ of adult population above 14 years with diabetes mellitus seen within the study period and using the hospital facility. Of the population, $2.8 \%$ were females while $47.2 \%$ were males. The newly diagnosed diabetic patients based on current WHO criteria (6) were only $5.6 \%$ of the study population. This clinic being a tertiary clinic, the patients who were referred as newly diagnosed were already on some form of glycaemialowering treatment for less than or equal to one month. Relating to their modes of glucose control, $22.3 \%$ were on insulin, $58.3 \%$ were maintained on oral hypoglycaemic agents, $9.2 \%$ were on combined insulin and oral hypoglycaemic agents while $4.6 \%$ were on diet-only as the only modality of glycaemic control. Most of these patients obtained their medication supplies from the hospital pharmacy, only occasionally did they supplement with their own purchases of the prescribed medication from the private chemists in town to bridge shortfalls of the hospitalsupplied medication. It is worth noting that the oral hypoglycaemic agents in use included mainly glibenclamide, chlorpropamide and metformin both in brand and their cheaper generics. The insulin available is the human lente and/or regular type of insulin (Novo Nordisk or Eli Lilly) brands. The lente insulin is in standard 100 Units vials.

The patients received their usual diabetes care, which included dietary advice, clinical evaluation and mid-morning random blood glucose assay. For the patients enrolled in the study, additional glycated haemoglobin was assayed by IMx boronic acid capture method with a non-diabetic range of $\mathrm{HbA1}$ c 4.4-6.4\%. The hospital does not routinely offer glycated haemoglobin assays because of its cost limitations.

The nutrition assistants on rota offered the dietary advice. Clinical evaluation was done by consultant physicians in diabetes care, post-graduate internists in training and medical officers. Most of the diabetes education was done by the nursing staff in the clinic. Blood glucose assay by glucose oxidase method was done by laboratory technicians/technologists within the clinic. The average number of clinic visits for most of these patients in one year is twice; more visits may be facilitated for patients with relatively more urgent needs.
A typical clinic visit involved weight, height and blood pressure measurements by the nurse. Patients then received nutritional advice and diabetes education either as a group or individually. Venous blood was taken for glucose assay followed by a 4-10 minutes consultation with the doctor, which involved review of blood pressure, glycaemia and a clinical examination of systems. Prescriptions were then generated for control of glycaemia, blood pressure or any other clinical situations that required medication.

\section{RESULTS}

Sample characteristics: The characteristics of the 305 patients who were included in the study are shown on Table 1. There was a female preponderance, at $52.8 \%$, however, this reflected not the national proportions but probable preferential attendance of the diabetic clinic. There were no significant differences in the means (SD) of BMI, age, duration of diabetes and glycaemic control between the male and female patients that were included in the study. The patients using oral agents to control their blood glucose represented over $65 \%$ of the population studied, which emphasised that type 2 diabetes mellitus was the more prevalent variety of the disease in the clinic, as seen also nationwide.

\section{Table 1}

Summary of the characteristics of the patients included in the study

\begin{tabular}{|c|c|}
\hline Characteristic & Population $(n=305)$ \\
\hline Socio-demographics & Mean (SD)/Proportion \\
\hline Males/females n (\%) & $144 / 161(47.2 / 52.8)$ \\
\hline \multirow[t]{2}{*}{ Age (years) } & Range: $14-92$ \\
\hline & Mean SD $52.54 \pm 13.35$ \\
\hline Body mass index $(\mathrm{BMI})\left(\mathrm{kg} / \mathrm{m}^{2}\right)$ & Range: $13.14-37.47$ \\
\hline Mean BMI for type 2 & Mean SD $26.05 \pm 4.37$ \\
\hline Type 1 n (\%) & $44(13.5 \%)$ \\
\hline Type 2 n $(\%)$ & $261(86.5 \%)$ \\
\hline \multicolumn{2}{|l|}{ Mode of glycaemic control } \\
\hline Insulin only & $68(22.3 \%)$ \\
\hline OHA only & $178(58.3 \%)$ \\
\hline OHA and insulin & $28(9.2 \%)$ \\
\hline Diet only & $14(4.6 \%)$ \\
\hline Newly diagnosed $\leq 2$ month (at inclusion) & $17(5.6 \%)$ \\
\hline \multirow[t]{2}{*}{ Glycated haemoglobin - (HbAl c) $(\%)$} & Range 4.46-17.07 \\
\hline & Mean \pm SD $8.91 \pm 2.75$ \\
\hline \multicolumn{2}{|l|}{ Blood pressure } \\
\hline Mean SBP (mmHg) & $141.6(26.8 \%)$ \\
\hline Mean DBP (mmHg) & $86.9(14.5 \%)$ \\
\hline
\end{tabular}

$\mathrm{OHA}=$ Oral Hypoglycaemic Agents

$\mathrm{SBP}=$ Systolic Blood Pressure

$\mathrm{DBP}=$ Diastolic Blood Pressure

Some of the newly diagnosed patients $(5.6 \%)$ were not on any treatment for glycaemic control at the time of enrollment to the study, however, their HbAlc results were included in the analysis of the total population means, but not in specific treatment categories. While HbAlc $\leq 7 \%$ remains the desired target of glycaemic control, in this study we used HbAlc $\leq 8 \%$ because it has been found to be the level achieved in conventionally treated diabetic patients. 
Figure 1

Glycaemic control and relative proportion of patients in the study

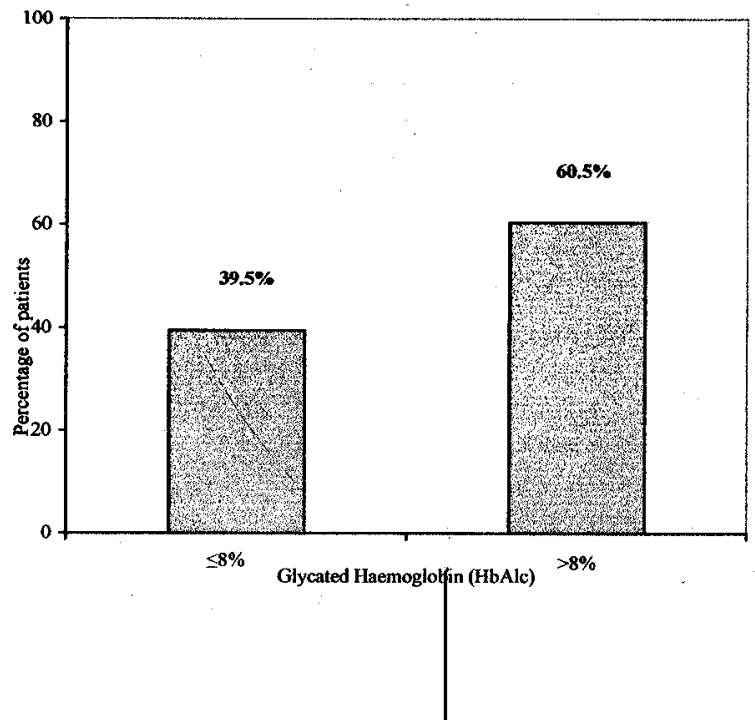

Only $39.5 \%$ of the study population were in relatively good glycaemic control (HbAlc $₫ 8.0 \%$ ). The rest, $60.5 \%$ had $\mathrm{HbAlc}>8.0 \%$ which is categ $\phi$ rised as poor glycaemic control (Figure 1). It is clear that the majority of the ambulatory diabetic patients using this outpatient clinic of the hospital had poor glycaemic control.

Figure 2

Patient characteristics and the level of glycated haemoglobin

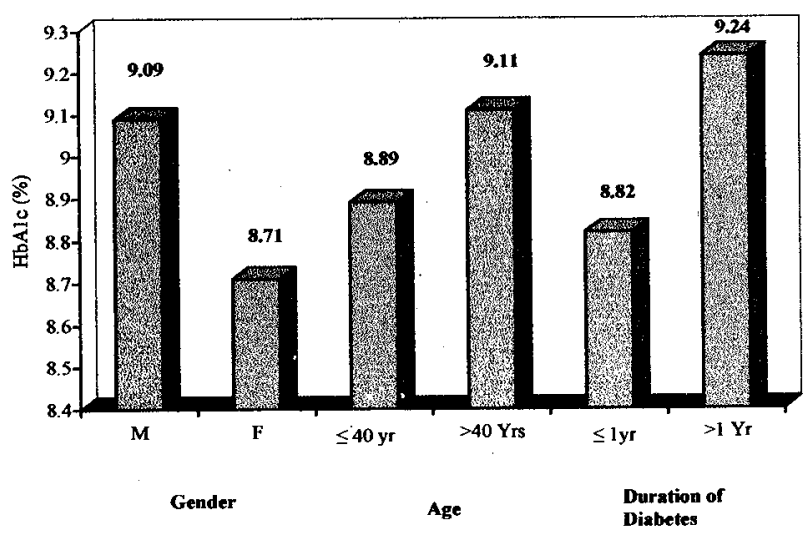

The gender, age of patients and their duration of diabetes had no statistically significant influences on their level of glycaemic control. This implies that other factors were responsible for the observed differences.
Figure 3

Mean Blood glucose of the patients by type of treatment

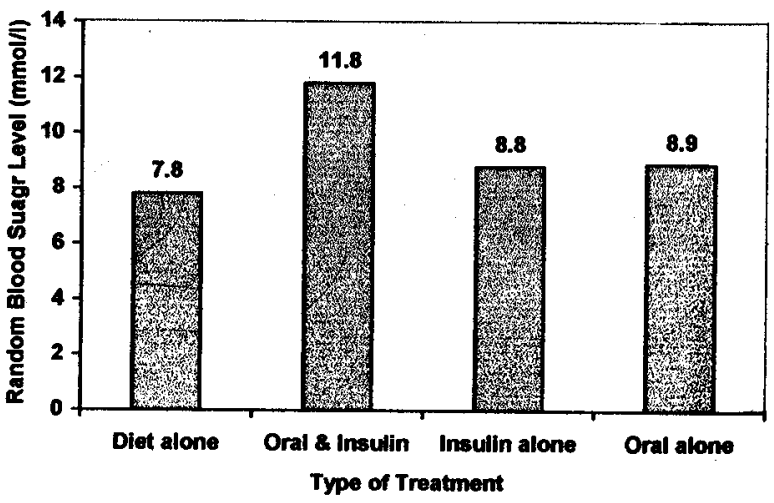

Figure 4

Mode of glycaemic control and the level of glycated haemoglobin

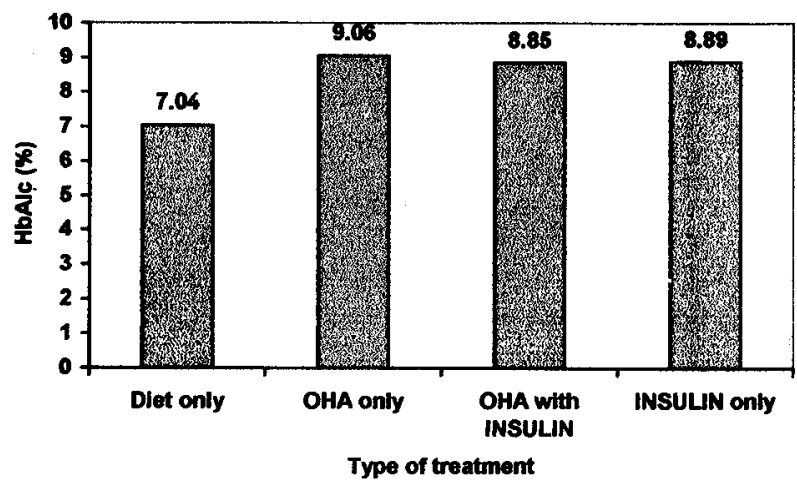

The patients who were on diet-only therapy for glycaemic control had the best control, mean $\mathrm{HbAlc}=$ $7.04 \%$ compared to the other modalities of lowering glycaemia namely, insulin only, OHA-only and combined $\mathrm{OHA}+$ insulin. The more plausible inference from this observation is that patients on diet-only therapy had relatively better beta cell functional reserve with fair endogenous insulin than patients on other alternative modes of glycaemic treatment. Good education per se is an unlikely main reason because all patients were handled by the same staff in the clinic.

Some other characteristics related to diabetes status of the study population were subjected to further analysis to establish which one(s) would be associated with poor glycaemic control of HbAlc above $8.0 \%$ (Table 2). 
Table 2

Unadjusted odds ratio for glycated $\mathrm{Hb} \geq 8 \%$ and some patient characteristics

\begin{tabular}{llcc}
\hline Gender & OR & 95\% CI & P-value \\
\hline Male & 1 & Reference & - \\
Female & 0.94 & $0.59-1.50$ & NS \\
Age of patients & & & \\
$\quad$ Less than 40 years & 1 & Reference & - \\
$\quad$ More than 40 years & 1.08 & $0.60-1.95$ & NS \\
$\quad \begin{array}{l}\text { Duration of Diabetes } \\
\quad \text { Less than I year }\end{array}$ & 1 & Reference & - \\
$\quad$ More than 1 year & 0.93 & $0.53-1.63$ & NS \\
$\quad$ Type of Treatment & & & \\
$\quad$ Insulin & 1 & Reference & - \\
$\quad$ Oral agents & 1.41 & $0.84-2.36$ & NS \\
$\quad$ Diet only & 0.24 & $0.07-0.79$ & 0.001 \\
$\quad$ & & & \\
$\quad$
\end{tabular}

The odds ratio (OR) of gender, age of the patients and their duration of diabetes were around unity (Table 2). This suggested that these characteristics were not impacting significantly on the level of glycaemic control of the study population. However, the patients on diet-only therapy were the least likely ( $\mathrm{OR}=0.24, \mathrm{p}<0.001$, significant) to have poor glycaemic control of HbAlc above $8.0 \%$. The patients on OHA-only were the most likely (OR $=1.41$, $\mathrm{p}>0.05)$ to have poor glycaemic control even though this did not attain statistically significant level. This observation may mean sub-optimal or inappropriate use of oral hypoglycaemic agents in the study population.

Linear regression of glycaemic control against duration of diabetes was done for only the patients on OHA-only therapy, which showed a rising trend of HbAlc. This could be interpreted as deteriorating beta cell function over time. However, the scatter of HbAlc values was quite marked which reflected the overall poor glycaemic control of the patients without regard to duration of diabetes.

\section{DISCUSSION}

There is a growing evidence that optimization of glycaemic control reduces the incidence of diabetes-related complications of retinopathy, neuropathy $(3,4,7)$. To attain optimal glycaemic control drug therapy, education and health care facilities need also be optimal. The study has shown that more than $60 \%$ of the ambulatory diabetes (type 1 and 2) using the hospital outpatient clinic do not attain the desired glycaemic level of control.

Diabetes care apparently is a challenge to both developed and developing countries alike. One Norwegian study (8) of type 2 diabetics in a general practice found fewer than $50 \%$ of the study population had good control. In an Ethiopian study (9), it was observed that $77 \%$ of their study population were in poor glycaemic control. While we recognise the difference in standards and test methods used in these studies relative to our study, it is sufficient to appreciate that a significant proportion of the patients in these centers did not attain the set levels that would put them in the category of good glycaemic control.
The challenges in diabetes care in both developing and developed countries may be similar in terms of disease course and burden but the capabilities to cope in resource allocation, expertise and health care facilities are very different and are certainly superior in the developed countries. Therefore, the observed high proportion of diabetic patients who are categorised as poorly controlled can be explained by several probable reasons. The supply of insulin and oral agents to the hospital may not be sustainable throughout the year to cater for all patients, needs. The patients in this study are a low-income group with major economic incapacitation. They largely depend on the hospital to cater for most of the requirements for their diabetes care.

This study also found that age, gender of patients and the duration of diabetes did not significantly influence their diabetes control. A similar observation was made in a Michigan community study of type 2 diabetic patients (10). This shows that there are other factors that are responsible for the levels of glycaemic control observed in this study. Erratic supply and high cost of insulin and its inaccessibility are perennial problems of developing countries $(11,12)$, Kenya included. These are likely the major reasons responsible for poor glycaemic control in diabetics who depend on insulin.

The likelihood of poor glycaemic control, HbA1c above $8.0 \%$ was lowest in diet-only therapy, possibly because these patients had better endogenous insulin production. The patients on OHA-only had the highest likelihood of poor glycaemic control. The probable reasons are various. Progressive beta cell failure is an established phenomenon in type 2 diabetes (13), a process that was certainly at play in these patients. Maybe a good number already needed insulin therapy at the time of the study. It was also possible that the oral agents were not optimal in doses either singly or in combination, quite a probable reason because of costs and provider-related factors. Lastly, but not least, the hospital where the study was conducted uses generics of oral agents, which are much cheaper than their branded products. Whether these generics have bioavailability and efficacy equal to the brands needs further investigation. It was observed during the study that some of the generic oral agents might be abetting poor glycaemic control.

In a population of type 2 diabetics who are perennially in poor glycaemic control, it is very difficult determining with some certainty when beta cell failure becomes absolute to warrant initiation of insulin therapy. This fact is enhanced by lack of good laboratory support in units that operate in a low-income environment, like our Kenyatta National Hospital.

To achieve and maintain good glycaemic control in type 2 diabetes mellitus, about $5-10 \%$ of these patients should be transferred to insulin therapy each year (14). It is also important to recognise that ultimately, most patients with type 2 diabetes mellitus will require treatment with insulin, either alone or in combination with an oral agent (15). Decision-making to select the best medication or 
combination of medications for management of hyperglycaemia in type 2 diabetes mellitus continues to reside, to a large degree, in the realm of "art". While there are relatively clear beneficial situations that dictate the use of one medication over another, as well as some obvious contraindications for all of the medications, often the choices are not clear cut (16). There is need for a more indepth investigation on reason(s) for lack of optimal glycaemic control among a substantial proportion of treated diabetic patients in this hospital. Elucidation of such variables that negatively impact on good glycaemic control, particularly in a low-income group like our study population, will provide a basis for an appropriate intervention in the population studied and similar populations elsewhere.

In conclusion, it should be remembered that in the developing countries, shortage of insulin supply and poor access to it remain part of major deterrents to good glycaemic control in the patients who depend on it. A beautiful commentary from Wallace and Mathew. (17) with an apt title, 'Poor glycaemic control in type 2 diabetes: a conspiracy of disease, sub-optimal therapy and attitude", is a fitting summary of the challenges in managing diabetes and poverty is an additional conspirator in this part of the world.

\section{ACKNOWLEDGEMENTS}

To the Director, Kenyatta National Hospital for permission to publish this manuscript. We are also indebted to the diabetes healthcare providers in the outpatient diabetes clinic of the hospital for their role in patient care. We are grateful to Emma for secretarial services.

\section{REFERENCES}

1. World Health Organization. In: "World Health Report. (Africa) conquering suffering, enriching humanity" World Health Organization, Geneva. 1997.

2. King, H.: Problems associated with the costs of diabetes care in Africa and Asia: a World Health Organization perspective. In: The Economics of Diabetes Care: An International perspective. Editors: Rhys Williams, Tuomilehto, J., Bjork, S., Blackwell Science. 2000; 61-68.
3. The Diabetes Control and Complications Trial Research Group.: The effect of intensive treatment of diabetes on the development and progression of long term complications in insulin-dependent diabetes mellitus. New. Eng. J. Med. 1993; 329:977-986.

4. UK Prospective Diabetes Study (UKPDS) Group.: Intensive blood glucose control with sulphonylureas or insulin compared with conventional treatment and risk of complications in patients with type 2 diabetes (UKPDS 33). Lancet. 1998; 352:837-853

5. Yearly records, Kenyatta National Hospital, 1997.

6. Alberti, K.G.M.M. and Zimmet, P.Z., for WHO consultation.: Definition, diagnosis and classification of diabetes mellitus and its complications. Part 1: Diagnosis and classification of diabetes mellitus. Provisional report of WHO consultation. Diabetes Med. 1998; 15:539-553.

7. Okhubo, Y., Kishikawa, H., Araki, E., et al. Intensive insulin therapy prevents the progression of diabetic microvascular complications in Japanese patients with NIDDM: A randomized prospective six-year study. Diabetes Res. Clin. Pract. 1995; 28:103-117.

8. Claudi, T., Cooper, J., Skogoy, K., et al. Diabetesomsorg, I morsk allmennpraksis - En tilstandsrapport fra salten og deler av Rogaland. Tidsskr Nor Loegeforen. 1997; 117:3661-3664.

9. Gebre-Yohannes, A., and Rahlenbeck, S. I. Glycaemic control and its determinants in diabetic patients in Ethiopia.DiabetesRes Clinical Pract. 1997; 35:129-134

10. Blaum, C.S., Velez, L., Hiss, R. G., and Halter J. B. Characteristics related to poor glycaemic control in NIDDM patients in community practice. Diabetes Care. 1997; 20: 7-10.

11. McLarty, D.G., Swai, A.B.M., and Alberti, K.G.M. M. Insulin availability in Africa an insoluble problem? Int. Diabetes Dig. 1994; 5:15-17.

12. Mbanya, J. C. Diabetes Care in Cameroon. Int. Diabetes Dig. 1993; 4:146-150.

13. U.K. Prospective Diabetes Study Group.: Overview of six years' therapy of type 2 diabetes. A progressive disease, UKPDS (16). Diabetes. 1995; 14:1249-1258.

14. Sonnaville, J.J.J., Snoek, K.F.J., Colly, L.P., et al. Well-being and symptoms in relation to insulin therapy in type 2 diabetes. Diabetes Care. 1998; 21:919-924.

15. DeFronzo, R.A. Pharmacologic therapy for type 2 diabetes mellitus - Review. Ann. Intern. Med. 1999; 131:286-303.

16. White, J. R. The pharmacological reduction of Blood Glucose in patients with type 2 diabetes mellitus. Clinical Diabetes. 1998; 16:58-67.

17. Wallace, T.M, and Mathews D.R. Poor glycaemic control in type 2 diabetes: A conspiracy of disease, sub-optimal therapy and attitude. Quart. J. Med. 2000; 93:369-374. 